\title{
Correlation of corticosterone elevation and negative contrast varies as a function of postshift day
}

\author{
CHARLES F. FLAHERTY, HOWARD C. BECKER, and LARISSA POHORECKY \\ Rutgers University, New Brunswick, New Jersey
}

\begin{abstract}
In three experiments, rats shifted from $32 \%$ to $4 \%$ sucrose consumed less of the $4 \%$ sucrose than did rats that had received only the $4 \%$ solution. Experiment 1 showed that this negative contrast effect was not accompanied by a corticosterone elevation on the first day subsequent to the shift. Experiments 2 and $2 a$ showed that corticosterone levels were substantially elevated on the 2nd postshift day and that there was a tendency for degree of elevation in corticosterone to be related to degree of lick suppression. These results are discussed in terms of other data suggesting that anxiolytic drugs and disinhibitory stimuli are more effective in alleviating contrast on the 2nd postshift day than on the 1st postshift day. It is suggested that, in the present paradigm, reaction to stimulus change may be the primary determinant of contrast on the 1st postshift day, but emotional processes related to reward loss and/or conflict develop by the 2nd postshift day.
\end{abstract}

Rats shifted from a $32 \%$ sucrose solution to a $4 \%$ sucrose solution consume substantially less of the $4 \%$ solution than animals that have experienced only $4 \%$ sucrose. This negative contrast effect in consummatory behavior occurs under a variety of experimental conditions and seems to parallel negative contrast obtained following downshifts in the amount of reward in instrumental tasks (Crespi, 1942; Flaherty, 1982; Vogel, Milkulka, \& Spear, 1968).

One of the traditional explanations of negative contrast effects that occur in the runway is that the reward downshift elicits a negative emotional response, such as frustration, which interferes with approaching the goal area. This interference results in slower running, which is measured as negative contrast (Amsel \& Stanton, 1980; Crespi, 1944; Flaherty, 1982; Spence, 1956; Stanton, Lobaugh, \& Amsel, 1984).

Does the negative contrast effect that occurs in the consumption of sucrose solutions also involve a negative emotional response? This is not necessarily the case, since the decline in lick frequency that occurs subsequent to the shift could reflect a neophobic or generalization decrement effect as the animals become adapted to a different solution. However, there are data which indicate a more active process. For example, presenting a novel stimulus (loud tone) during the postshift period had a small, but reliable, effect of decreasing the size of the negative con-

This research was supported by grants from the Charles and Johanna Busch Memorial Fund and by Grant BNS 83-02838 from the National Science Foundation to C.F. and Grant K02AA 000 45-09 from the National Institute of Alcohol Abuse and Alcoholism to L.P.

L. Pohorecky is associated with the Center for Alcohol Studies at Rutgers University. The mailing address of the other two authors is: Department of Psychology, Busch Campus, Rutgers University, New Brunswick, NJ 08903. trast (Lombardi \& Flaherty, 1978). An interpretation of contrast in terms of generalization decrement would have to predict that the addition of the tone would enhance negative contrast, since the novel tone would be compounded with the novel postshift sucrose solution. Since the tone did not enhance the lick rate of unshifted $4 \%$ control animals or the lick rate of a $2 \%$ group (which licked at about the same rate as the shifted $4 \%$ animals), it would seem that the tone had a disinhibitory effect on the shifted animals and not a simple energizing effect on the lick rate of all animals.

The effects of a variety of drugs on consummatory contrast would also suggest the involvement of an emotional response and active inhibition of licking in the shifted animals. For example, negative contrast is essentially eliminated by the appropriate dose of the tranquilizers chlordiazepoxide and midazolam (Becker, 1984; Becker \& Flaherty, 1983; Flaherty, Lombardi, Wrightson, \& Deptula, 1980) and it is reliably reduced by ethanol (Becker \& Flaherty, 1983) and sodium amobarbital (Flaherty \& Driscoll, 1980; Flaherty, Becker \& Driscoll, 1982). Furthermore, relatively ineffective doses of chlordiazepoxide and ethanol have an additive effect in reducing contrast when administered together (Becker \& Flaherty, 1983). Drugs that do not have anxiolytic effects in humans have thus far been found to be ineffective in reducing consummatory contrast. For example, contrast is not reduced by the anticholinergic scopolamine (Becker, 1984; Flaherty \& Meinrath, 1979), the antihistamine pyrilamine (Becker, 1984), the antiserotonergic drug methysergide (Becker, 1984), or the neuroleptics haloperidol and chlorpromazine (unpublished data of Flaherty \& Becker).

Since the drugs that are effective in reducing contrast have anxiolytic action in humans, these data are consis- 
tent with a role for an emotional response in the occurrence of consummatory contrast. Other data indicate that a downshift in the concentration of sucrose solutions is aversive to rats. For example, Daly (1974) has shown that rats will learn a response to escape from a stimulus that had been paired with a downshift in sucrose concentration. Downshifting sucrose concentration also leads to an increase in rearing and ambulatory behaviors in an openfield situation (Flaherty, Blitzer, \& Collier, 1978; Flaherty, Troncoso, \& Deschu, 1979), a pattern of behavior that has been associated with emotionality (Royce, Poley, \& Yeudall, 1973). Finally, it has been shown that lesions of the basolateral amygdala reduce consummatory negative contrast and lesions of the corticomedial aspects of the amygdala eliminate negative contrast (Becker, Jarvis, Wagner, \& Flaherty, 1984). The amygdala has been implicated in a variety of emotional behaviors, and lesioning the amygdala has a taming effect on animals (e.g., Isaacson, 1974).

Thus, there is substantial circumstantial evidence suggesting that an emotional response is causally related to the occurrence of negative contrast in consummatory behavior. The present paper is concerned with another possible indicant of emotionality-the secretion of corticosterone. Most stressors enhance the activity of the pituitary-adrenal system (Axelrod \& Reisine, 1984; Miller, 1980), and corticosteroid levels often correlate with performance in tasks thought to involve emotionality (e.g., Coover, Sutton, Welle, \& Hart, 1978; Coover, Murison, Sundberg, Jellestad, \& Ursin, 1984; Overmier, Patterson, \& Wielkiewicz, 1980; Weinberg \& Levine, 1980). There is also evidence that a loss of reward or a reduction in reward will elevate corticosteroid levels. For example, shifting rats or pigs to extinction in instrumental tasks leads to an elevation in corticosteroids (Dantzer, Arnone, \& Mormede, 1980; Goldman, Coover, \& Levine, 1973), and extinction may be thought of as the limiting case of negative contrast (Gonzalez, Fernhoff, \& David, 1973). It has also been found that a shift to a reduced frequency of reinforcement will lead to elevated corticosteroid levels (Goldman et al., 1973). Thus, there is reason to expect that downshifts in sucrose concentration, if such downshifts are aversive, will lead to an increase in pituitary-adrenal activity. However, such an outcome is not a certainty, since all of the above-cited studies were concerned with instrumental behavior and the contrast paradigm under consideration here involves consummatory behavior. Furthermore, there is a peculiarity in this consummatory contrast that has not yet been mentioned. That is, some of the evidence indicating the involvement of an emotional response is more persuasive on the 2 nd postshift day than on the 1st postshift day. For example, the disinhibition effect described above was found on the 2nd and subsequent postshift days, but not on the 1st postshift day. Similarly, ethanol and chlordiazepoxide are without effect on the 1st postshift day, but have potent effects on the 2 nd postshift day.
What could account for these differences? One possibility is that the mechanisms producing contrast change over the postshift period (Flaherty et al., 1980). That is, contrast may initially be related to a generalization decrement or a neophobic response as the animal detects a difference between the current solution and its memory of the previously experienced solution. This comparison process may then yield an evaluative judgment in which the hedonic value of the postshift solution is found to be less than that of the memory of the preshift solution. As a result of this evaluation, the animal may be in a conflict situation-drawn to the $4 \%$ postshift solution because of its absolute rewarding properties but also repelled by this solution because it is relatively less rewarding than the $32 \%$ preshift solution. It is possible that this conflict phase does not develop until the 2 nd postshift day, and it is during conflict that the novel stimulus is disinhibitory and the anxiolytic drugs are particularly effective. This explanation, though complex, is consistent with recent developmental literature postulating the sequential unfolding of differential mood or emotional states (e.g., Malatesta \& Haviland, 1982; Sackett, 1979).

There is a simpler explanation for the differential effects of the anxiolytics and disinhibitory stimulus across the postshift period. Contrast is usually largest on the 1st postshift day and then diminishes in a systematic fashion over subsequent days until, usually by the 3 rd or 4 th postshift day, contrast is no longer present. It is possible that the drugs are differentially effective on the first 2 postshift days because contrast is so robust on the 1st postshift day that it overrides the effects of the drugs. On the 2nd postshift day, contrast may be weaker, perhaps because of the now more remote comparison with the memory of the preshift solution (Spear, 1967) and/or because of the experience with the postshift solution. It may be when contrast is thus weakened that the drugs become effective.

The assay of corticosterone levels that is the subject of this paper will provide evidence concerning the involvement of stress/emotionality in contrast per se and evidence relevant to the two hypotheses regarding the differential effectiveness of chlordiazepoxide, ethanol, and the disinhibitory stimulus on the $1 \mathrm{st}$ and 2 nd postshift days. If stress or emotionality did not develop in the consummatory contrast procedure until the 2 nd postshift day, then we would expect corticosterone levels to be greater on the 2nd than on the 1st postshift day. However, if the treatments were ineffective because contrast was more robust on the 1st postshift day than on the 2nd postshift day, then we would expect corticosterone levels to be greater on the 1st postshift day.

\section{EXPERIMENT 1}

This experiment was concerned with contrast and corticosterone response on the 1st postshift day. Three groups of rats were used; one group was given $32 \%$ sucrose in 
the preshift period and then shifted to $4 \%$ sucrose, the second group received $4 \%$ sucrose throughout the experiment, and the third group received $2 \%$ sucrose throughout the experiment. The latter group was included to control for the possibility that lick rate per se would have an effect on corticosteroid levels. Previous experiments had indicated that a group given access to $2 \%$ sucrose licks at approximately the same rate as a group shifted from $32 \%$ to $4 \%$ sucrose.

\section{Method}

Subjects. Twelve male Sprague-Dawley-derived rats, weighing approximately $350 \mathrm{~g}$, were used as subjects. The rats were individually housed in metal cages and maintained at $82 \%$ of their freefeeding weights by once-per-day feeding. Water was always available in the home cage. A 14-h/10-h light/dark cycle was in effect in the colony room, and the animals were tested each day approximately $3 \mathrm{~h}$ into the light phase of the cycle.

Apparatus. The subjects were tested in identical metal housing cages $(24.5 \times 17.5 \times 18 \mathrm{~cm})$ with wooden covers. A centrally located hole (1-cm diam) $7 \mathrm{~cm}$ above the floor was present in one wall of the cage. A graduated cylinder was placed outside the chamber such that the orifice of the drinking spout was centered in the hole and flush with the outside wall of the cage. A contact relay circuit was used to monitor licks, which were recorded on a microprocessor. The apparatus was located in a dimly illuminated room with white noise present during testing.

Procedure. The rats were assigned randomly to three groups $(n=4)$. One group received access to $32 \%$ sucrose for 10 days and then $4 \%$ sucrose for 1 additional day (shifted group). A second group was given access to $4 \%$ sucrose for all 11 days (unshifted controls), and a third group received $2 \%$ sucrose for all 11 days. The latter group was included to control for lick rate and volume consumed on the shift day. The 11 daily sessions consisted of a 5 -min access period to the appropriate sugar solution each day. The 5 -min period was timed from the first lick. The sugar solutions were mixed from commercial cane sugar and tap water by weight [sucrose/(sucrose + water)].

The animals were run in squads of three, one animal from each group. On each day, the animals were weighed and then wheeled into the testing room in wooden carrying cases mounted on a cart. At the end of the session, they were carried individually back to the colony room. The animals were fed their daily ration approximately $1 \mathrm{~h}$ after each daily session. Immediately following the conclusion of testing on the first postshift day (Day 11 of training), the animals were carried, one at a time, to a different room where they were decapitated and trunk blood was collected on ice. The samples were centrifuged and the serum was frozen and stored at $-20^{\circ} \mathrm{C}$ for later analysis. Serum corticosterone was assayed by the fluorometric method of Glick, von Redlich, and Levine (1964).

\section{Results and Discussion}

Animals downshifted from $32 \%$ to $4 \%$ sucrose showed a reliable negative contrast effect, but there was no correlated elevation in corticosterone levels. These results are shown in Figure 1. The left-hand portion of the figure shows terminal preshift lick frequency (Day 10) and lick frequency on the postshift day for all groups. A reliable sucrose $X$ day interaction $[F(2,9)=22.83, p<.001]$ from the analysis of variance indicated the following pattern. On the terminal preshift day, the $32 \%$ and $4 \%$ groups licked at the same frequency, both reliably higher than the $2 \%$ group [least significant difference (LSD) test, $p<.05]$. On the postshift day, the group shifted from

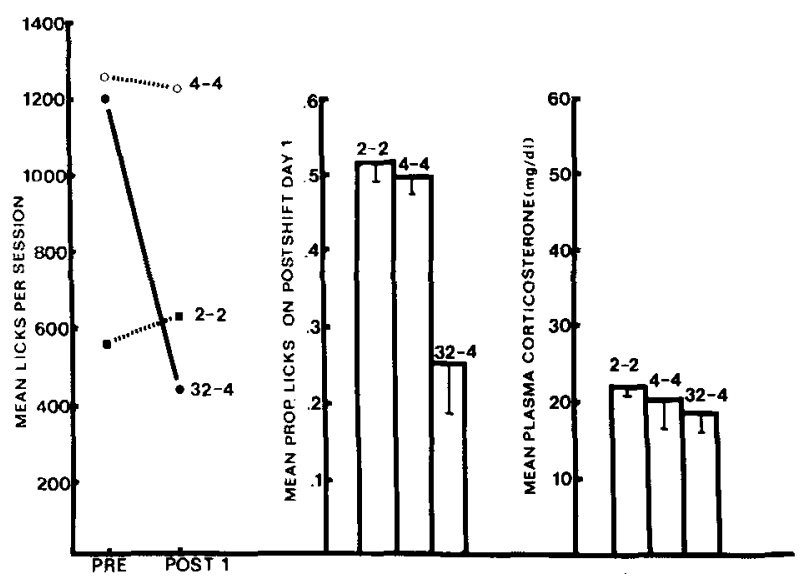

Figure 1. (Left panel) Mean licks per session on last preshift and first postshift day. (Center panel) Proportion licks [postshift/(postshift + last preshift)] for each group. (Right panel) Mean corticosterone level on first postshift day as a function of sucrose condition.

$32 \%$ to $4 \%$ sucrose licked reliably less than the unshifted $4 \%$ control and at a level not different from that of the $2 \%$ group (LSD test).

The middle panel of the figure presents the postshift lick data in terms of proportion of preshift lick frequency [Day 11/(Day $10+$ Day 11)]. These data show that the shifted animals licked proportionately less sucrose than either the $4 \%$ or $2 \%$ control groups, which did not differ $[F(2,9)=13.75, \mathrm{p}<.002]$.

The right-hand panel presents the corticosterone levels obtained on the postshift day. The three groups did not differ in terms of corticosterone levels $(F<1.00)$. Thus, the occurrence of a substantial contrast effect in consummatory behavior was not accompanied by activation of the pituitary-adrenal system.

\section{EXPERIMENT 2}

In this experiment, the animals were continued to the 2nd postshift day before being sacrificed for corticosterone analysis.

\section{Method}

Subjects. Eighteen naive male Sprague-Dawley-derived rats were used as subjects (six animals in each of three groups). The animals were maintained and housed as in Experiment 1 .

Apparatus. The apparatus was the same as that used in Experiment 1 .

Procedure. The procedure was the same as that used in Experiment 1 except that the animals were run for 2 postshift days instead of the single postshift day used in the first experiment.

\section{Results and Discussion}

The shifted rats showed a reliable negative contrast effect on both postshift days and the corticosterone levels measured following the 2nd postshift day were substantially elevated in the shifted rats, relative to the unshifted $4 \%$ controls and $2 \%$ controls. 


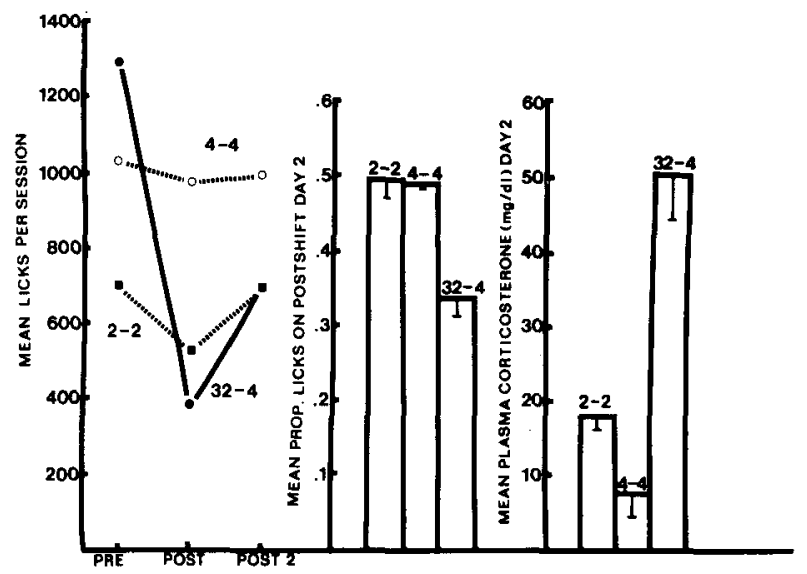

Figure 2. (Left panel) Mean licks per session on last preshift day and first 2 postshift days. (Center panel) Proportion licks [Day 10/Day 10 + Day 12)] on second postshift day. (Right panel) Mean corticosterone level on second postshift day as a function of sucrose condition.

The left-hand portion of Figure 2 shows lick frequencies in the three groups obtained on the terminal preshift day and on the 2 postshift days. These data do not include four animals from the $4 \%$ control group who were dropped from the experiment for failure to lick the sucrose in the preshift period. Analysis of variance indicated a reliable groups $\times$ days interaction $[F(4,22)=$ $21.76, \mathrm{p}<.001]$.

Subsequent analysis with the LSD test $(p<.05)$ indicated the following pattern of results. On the last preshift day, the $32 \%$ group licked more than the $4 \%$ group, which licked more than the $2 \%$ group. On the 1st postshift day, the shifted group licked less than the unshifted $4 \%$ controls and was not different in lick frequency from the $2 \%$ control group. The $2 \%$ control group also licked at a lower rate than the $4 \%$ group. On the 2 nd postshift day, the same pattern of results held: The shifted animals were reliably below the unshifted controls and were licking at essentially the same rate as the $2 \%$ group.

The middle panel of Figure 2 presents the proportion of licks [Day 12/(Day $10+$ Day 12)] made on the 2nd postshift day by each group relative to their terminal preshift lick levels. Analysis indicated reliable group differences $[F(2,11)=10.56, p<.003]$, and LSD tests indicated that the shifted animals were reliably below the two control groups.

The third panel of Figure 2 presents the corticosterone data. Analysis of these data indicated reliable group differences $[F(2,11)=21.05, p<.0003]$, and subsequent LSD tests showed that the corticosterone levels of the shifted group were reliably higher than those of the $4 \%$ and $2 \%$ groups, which did not differ.

Thus, the results of this experiment suggest that negative contrast becomes stressful on the 2 nd postshift day. Since, however, there were only two animals in the $4 \%$ control group, it was decided to replicate the main portion of the study.

\section{EXPERIMENT 2a}

This experiment replicated the procedure of Experiment 2 except that only the $32 \%$ and $4 \%$ groups were used-the $2 \%$ control group was not included.

\section{Method}

Subjects. The subjects were eight (four per group) naive male Sprague-Dawley-derived rats maintained as in the first two experiments.

Apparatus. The apparatus was the same as that used in the first two experiments.

Procedure. The procedure was the same as that used in Experiment 2, except that the $2 \%$ control group was not included.

\section{Results and Discussion}

As in Experiment 2, the shifted animals showed a reliable negative contrast effect and a reliable elevation in corticosterone levels on the 2 nd postshift day. The left panel of Figure 3 illustrates the lick frequencies obtained on the last preshift day and on the 2 postshift days for the shifted and unshifted groups. Analysis of variance indicated a reliable groups $X$ day interaction $[F(2,12)=30.33, p$ $<.001]$. Subsequent analysis with the LSD test indicated the following pattern of results. The $32 \%$ group was not licking at a reliably higher rate than the $4 \%$ group on the last preshift day, but their lick rate dropped to a level reliably below that of this group on both the 1st and 2nd postshift days. In addition, the small increase in lick rate shown by the shifted group between the 1 st and 2 nd postshift days was reliable.

The middle panel of Figure 3 presents the Day 2 postshift lick rate represented as a proportion of the terminal preshift lick rate. Analysis of variance showed that the shifted group licked proportionately less than the unshifted $4 \%$ group $[\mathrm{F}(1,6)=28.95, \mathrm{p}<.002]$.

The third panel of Figure 3 presents the corticosterone levels obtained on the 2 nd postshift day. The shifted group

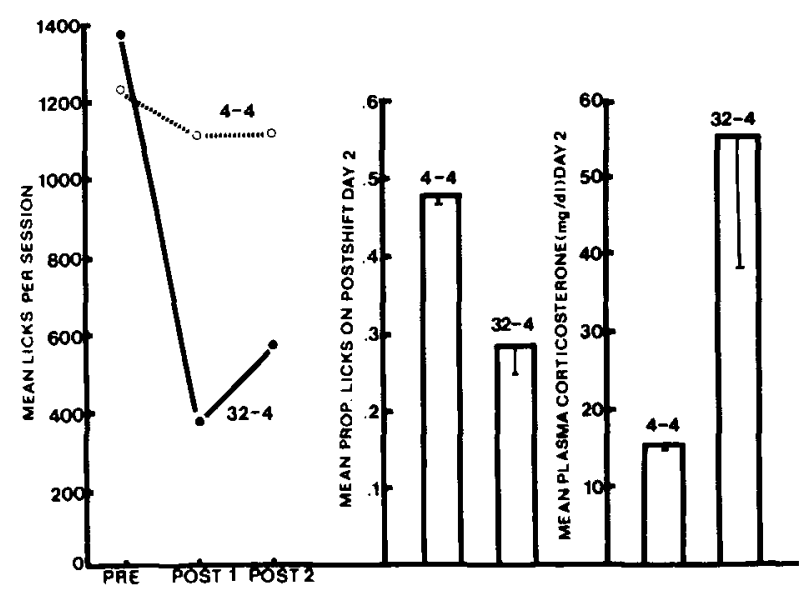

Figure 3. (Left panel) Mean licks per session on last preshift day and first 2 postshift days. (Center panel) Proportion licks [Day 10/Day 10 + Day 12)] on second postshift day. (Right panel) Mean corticosterone level on second postshift day as a function of sucrose condition. 
showed substantially greater corticosterone levels than the unshifted group and also was substantially more variable than the unshifted group. Since Cochran's C test showed that the variance between the two groups was significantly heterogeneous $(\mathrm{C}=0.9392, \mathrm{p}<.01)$ and since the sample size was small, a nonparametric test of significance was used for the corticosterone levels. The Mann-Whitney $\mathrm{U}$ test indicated that the shifted group showed reliably higher corticosterone levels than the unshifted group $(\mathrm{U}=\mathrm{O}, \mathrm{p}=.028$, two-tail).

Thus, the results of this experiment replicate those of Experiment 2 in finding an elevated corticosterone release correlated with the occurrence of negative contrast on the 2nd postshift day. Inspection of the data from Experiments 2 and $2 a$ also suggested that shifted animals that showed the greatest lick suppression had the highest levels of corticosterone. However, the correlation $(r=-.59)$ between proportion of licks made on postshift Day 2 and corticosterone levels in the two experiments combined failed to reach statistical reliability $(p=.073)$.

\section{GENERAL DISCUSSION}

The results of these three experiments show that substantial negative contrast effects occur in consummatory behavior on both the 1st and 2 nd day following a shift from $32 \%$ to $4 \%$ sucrose. However, an elevation in corticosterone level occurs only on the 2 nd postshift day. The data also suggested a possible relationship between degree of lick suppression on the 2 nd postshift day and degree of corticosterone elevation.

Although these results seem clear, one potentially bothersome factor is the lack of a reliable difference in preshift lick rates between the $32 \%$ and $4 \%$ groups in Experiment 1 . It is, however, unlikely that this effect is related to the difference between Experiment 1 and Experiments 2 and $2 a$ in corticosterone elevations. In general, rats show substantial variability in their lick rates for $4 \%$ sucrose, and whether or not a reliable difference is obtained over a 10-day preshift period depends upon the particular sample of rats in an experiment. The failure to obtain a difference in preshift lick rate does not seem to be related to degree of contrast when animals are shifted from $32 \%$ to $4 \%$ sucrose. For example, the proportion of preshift lick rate obtained on the 1st postshift day in the three experiments was as follows: 0.250 in Experiment $1,0.220$ in Experiment 2, and 0.210 in Experiment 2a. These differences are not reliable $(F<1.00)$. Although a significant difference in preshift lick rate was found only in Experiment 2, we found elevated steriod levels in the shifted animals in both Experiment 2 and Experiment $2 \mathrm{a}$. Thus, we accept the results as indicating that steriods are elevated on the 2 nd day of contrast but not on the 1st day.

The results obtained in these experiments are consistent with other data mentioned in the introduction. That is, the presentation of a disinhibitory stimulus is effective on the 2 nd but not on the 1 st postshift day. Also, the anxiolytics chlordiazepoxide and ethanol are effective in reducing contrast on the 2 nd postshift day but are without effect on the 1st postshift day. Taken together, these data imply that the processes related to negative contrast change over the postshift period. In particular, it would appear that contrast does not become stressful until the 2nd postshift day in the paradigm employed in these experiments. These corticosterone data favor one of the two alternative hypotheses described in the introduction. The occurrence of a corticosterone elevation on the 2 nd but not on the 1st postshift day indicates that the failure of CDP and ethanol to be effective on the 1st postshift day is not due to the fact that contrast is more robust on the 1 st postshift day. Instead, these differences may be related to the fact that an emotional mechanism related to incentive loss or conflict may be the principal causal agent operating on the 2nd postshift day but some other process, such as generalization decrement, may be primarily responsible for contrast on the 1st postshift day.

A detailed consideration of how these data relate to the contrast that occurs in runway situations is not appropriate at the present time since analogous Day 1 versus Day 2 drug or corticosterone studies seem not to have been done in that situation and, in addition, a large number of studies have shown that successive contrast does not occur following the downshift of sucrose concentration in the runway (see Flaherty, 1982, for a review of these issues). It should be noted, though, that Capaldi (1972) has suggested that generalization decrement and frustration both contribute to negative contrast in the runway when number of food pellets is decreased and that anxiolytics do diminish the degree of negative contrast obtained in such situations (Rosen \& Tessel, 1970).

All of these data suggest that the negative contrast procedure may involve mechanisms in the rat that could be considered analogous to disappointment in humansa reward expectancy, the failure of that expectancy to be confirmed, a stress response, and the apparent relief of that stress by anxiolytics (cf. Coover, 1982; Flaherty, 1982; Klinger, 1975).

It remains to be determined how long the corticosterone elevation endures following a downshift in reward, whether or not such an elevation may occur on the 1st postshift day if measurements are obtained at a time later than approximately $5 \mathrm{~min}$ after the first experience with the shift, the effects of the corticosterone elevation following contrast on other systems such as the immune system, the relationship of corticosterone changes to positive contrast in this paradigm, and whether or not a corticosterone elevation will occur in animals that are repeatedly downshifted (cf. Flaherty, Becker, \& Checke, 1983).

\section{REFERENCES}

AmSEl, A., \& Stanton, M. (1980). Ontogeny and phylogeny of paradoxical reward effects. In J. S. Rosenblatt, R. A. Hind, C. Beer, \& M. Busnel (Eds.), Advances in the study of behavior (pp. 227-274). New York: Academic Press. 
AXElRod, J., \& Reisine, T. D. (1984). Stress hormones: Their interaction and regulation. Science, 224, 452-459.

BECKER, H. C. (1984). Effects of midazolam and three serotonin antagonists on consummatory negative contrast. Unpublished doctoral dissertation, Rutgers University.

Becker, H. C., \& FlaherTy, C. F. (1983). Chlordiazepoxide and ethanol additively reduce gustatory negative contrast. Psychopharmacology, 80, 35-37.

Becker, H. C., JARvis, M., Wagner, G., \& Flaherty, C. F. (1984). Medial and lateral amygdala lesions differentially influence contrast with sucrose solutions. Physiology \& Behavior, 33, 707-712.

CAPALDI, E. J. (1972). Successive negative contrast effect: Intertrial interval, type of shift, and four sources of generalization decrement. Journal of Experimental Psychology, 96, 433-438.

CoOver, G. D. (1982). Positive and negative expectancies: The rat's reward environment and pituitary adrenal activity. In $\mathrm{H}$. Ursin \& $\mathbf{R}$. Murison (Eds.), Advances in the biosciences: Vol. 42. Biological and psychological basis of psychosomatic disease (pp. 45-60). Oxford: Pergamon Press.

Coover, G. D., Murison, R., Sundberg, H., Jellestad, F., \& URSIN, H. (1984). Plasma corticosterone and meal expectancy in rats: Effects of low probability cues. Physiology \& Behavior, 33, 179-184.

Coover, G. D., Sutton, B. R., Welle, S. L., \& HART, R. P. (1978). Corticosterone responses, hurdle-jump acquisition, and the effects of dexamethasone using classical conditioning of fear. Hormones \& Behavior, 11, 279-294.

Crespi, L. P. (1942). Quantitative variation of incentive and performance in the white rat. American Journal of Psychology, 55, 467-517.

CresPi, L. P. (1944). Amount of reinforcement and level of performance. Psychological Review, 51, 341-347.

DALY, H. B. (1974). Reinforcing properties of escape from frustration. In G. H. Bower (Ed.), The psychology of learning and motivation. New York: Academic Press.

Dantzer, R., ARnone, M., \& Mormede, P. (1980). Effects of frustration on behavior and plasma corticosteroid levels in pigs. Physiology \& Behavior, 24, 1-4.

FlaherTy, C. F. (1982). Incentive contrast: A review of behavioral changes following shifts in reward. Animal Leaming \& Behavior, 10, 409-440.

Flaherty, C. F., BeCKer, H. C., \& CHECKe, S. (1983). Repeated successive contrast in consummatory behavior with repeated shifts in sucrose solution. Animal Learning \& Behavior, 11, 407-414.

Flaherty, C. F., Becker, H. C., \& Driscoll, C. (1982). Conditions under which amobarbital sodium influences consummatory contrast. Physiological Psychology, 10, 122-128.

Flaherty, C. F., Blitzer, R., \& Collier, G. H. (1978). Open field behaviors elicited by reward reduction. American Journal of Psychology, 91, 429-443.

Flaherty, C. F., \& Driscoll, C. (1980). Amobarbital sodium reduces successive gustatory contrast. Psychopharmacology, 69, 161-162.

Flaherty, C. F., Lombard, B. R., Wrightson, J., \& Deptula, D. (1980). Conditions under which chlordiazepoxide influences gustatory contrast. Psychopharmacology, 67, 269-277.

Flaherty, C. F., \& Meinrath, A. B. (1979). Influence of scopolamine on sucrose intake under absolute and relative test conditions. Physiological Psychology, 7, 412-418.
Flaherty, C. F., Troncoso, B., \& Deschu, N. (1979). Open field behaviors correlated with reward availability and reward shift in three rat strains. American Joumal of Psychology, 92, 385-400.

Glick, D., von Redlich, D., Levine, S. (1964). Fluorometric determination of corticosterone and cortisol in 0.02-0.05 milliliters of plasma or submilligram samples of adrenal tissue. Endocrinology, 74, 653-655.

Goldman, L., Coover, G. D., \& Levine, S. (1973). Bidirectional effects of reinforcement shifts on pituitary adrenal activity. Physiology \& Behavior, 10, 209-214.

Gonzalez, R. C., Fernhoff, D., \& David, E. G. (1973). Contrast, resistance to extinction, and forgetting in rats. Journal of Comparative \& Physiological Psychology, 84, 562-571.

IsAacson, R. L. (1974). The limbic system. New York: Plenum Press. KLINGER, E. (1975). Consequences of commitment to and disengagement from incentives. Psychological Review, 82, 1-25.

LOMBARDI, B. R., \& Flaherty, C. F. (1978). Apparent disinhibition of successive but not of simultaneous negative contrast. Animal Learming \& Behavior, 6, 30-42.

Malatesta, C. Z., \& Haviland, J. M. (1982). Learning display rules: The socialization of emotion expression in infancy. Child Development, 53, 991-1003.

Miller, N. E. (1980). A perspective on the effects of stress and coping on disease and health. In S. Levine \& H. Ursin (Eds.), Coping and health. New York: Plenum Press.

Overmier, B., Patterson, J., \& Wielkiewicz, R. M. (1980). Environmental contingencies as sources of stress in animals. In S. Levine \& H. Ursin (Eds.), Coping and health. New York: Plenum Press.

Rosen, A. J., \& TESSEL, R. E. (1970). Chlorpromazine, chlordiazepoxide, and incentive shift performance in the rat. Journal of Comparative \& Physiological Psychology, 72, 257-262.

Royce, J. R., Poley, W., \& Yeudall, L. T. (1973). Behavior-genetic analysis of mouse emotionality: I. Factor analysis. Journal of Comparative \& Physiological Psychology, 83, 36-47.

SACKETT, G. P. (1979). The lag sequential analysis of contingency and cyclicity in behavioral interaction research. In J. D. Osofsky (Ed.), Handbook of infant development. New York: Wiley.

SPENCE, K. W. (1956). Behavior theory and conditioning. New Haven: Yale University Press.

Stanton, M., Lobaugh, N., \& AmSEl, A. (1984). Age of first appearance of simultaneous and successive negative contrast in infant rats. Joumal of Experimental Psychology: Animal Behavior Processes, 10, 376-389.

SPEAR, N. E. (1967). Retention of reinforcer magnitude. Psychological Review, 74, 216-234.

Vogel, J. R., MikulKa, P. J., \& Spear, N. E. (1968). Effects of shifts in sucrose and saccharin concentrations on licking behavior in the rat. Journal of Comparative \& Physiological Psychology, 66, 661-666.

WeINBERG \& LE VINe (1980). Psychobiology of coping in animals: The effects of predictability. In S. Levine \& H. Ursin (Eds.), Coping and health. New York: Plenum Press, 1980.

(Manuscript received February 28, 1985; revision accepted for publication July 22,1985 .) 